\title{
Quantum group symmetry of integrable models on the half-line
}

\author{
Gustav W Delius* and Alan George \\ Department of Mathematics, The University of York, York YO10 5DD,UK \\ E-mail: 'gwd2@york. ac.uki and ag160@york.ac.uk
}

ABstract: We derive the non-local conserved charges in the sine-Gordon model and affine Toda field theories on the half-line. They generate new kinds of symmetry algebras that are coideals of the usual quantum groups. We show how intertwiners of tensor product representations of these algebras lead to solutions of the reflection equation. We describe how this method for finding solutions to the reflection equation parallels the previously know method of using intertwiners of quantum groups to find solutions to the Yang-Baxter equation.

\section{Introduction}

During the last year the search for the quantum group symmetry of integrable quantum field theories with boundaries has finally succeeded ${ }^{1}$. MacKay and Short in their contribution to these proceedings [1] on the half-line. In this contribution we present the non-local charges of the sine-Gordon model and affine Toda field theories on the half-line.

These non-local charges generate symmetry algebras of a very unusual kind. In contrast to all other known symmetry algebras they are not bialgebras. We will refer to them as boundary quantum groups. They are coideals of the usual quantum groups. Just as quantum groups lead to solutions of the Yang-Baxter equation, our new boundary quantum groups lead to solutions of the reflection equation, also known as the boundary Yang-Baxter equation.

The Yang-Baxter equation appears throughout $1+1$ dimensional integrable systems in two roles. Firstly to define commuting transfer matrices one requires L operators that satisfy the Yang-Baxter equation [2]. Secondly particles scatter in a factorizable way, the consistency condition on the factorization is given by the Yang-Baxter equation [2] ${ }_{2} \overline{3}$.

${ }^{*}$ Speaker.

${ }^{1}$ Earlier calculations for the sine-Gordon model at the free fermion point were done in [1] 
In section $2 \overline{2}_{1}^{2}$ we will review the Yang-Baxter equation in the specific context of particle scattering and we will recall how quantum group symmetry provides an easy way to find its solutions [1] $\overline{1} \overline{3}$ in].

In order to restrict the domain of an integrable system to the half line or an interval one must use solutions of the reflection equation in addition to solutions of the YangBaxter equation. Such reflection matrices are used to describe factorizable scattering of particles from the boundary $\left[\bar{p}_{1}^{\prime}, \overline{1} \overline{2}\right]$. Reflection matrices are also needed for the definition of commuting transfer matrices [2] $\left.{ }_{2}^{1}\right]$. We describe the reflection equation in section emphasizing how it parallels the Yang-Baxter equation. We show how boundary quantum groups allow us to find solutions of the reflection equation by simply solving a linear intertwining equation.

In section 舀 we briefly review the non-local charges of the sine-Gordon model [3] that generate the affine algebra $U_{q}\left(\widehat{s l_{2}}\right)$. We then show how the generators of the boundary quantum groups for the sine-Gordon model restricted to the half-line by a general integrable boundary condition can be constructed from these by using first order perturbation theory [i]. These boundary quantum groups have been used to determine $s l_{2}$ reflection matrices

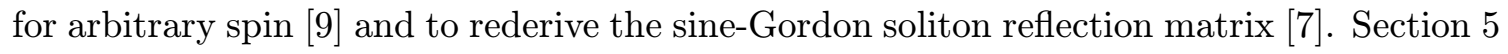
generalizes the calculation to affine Toda theories on the half-line lini, where it has been used

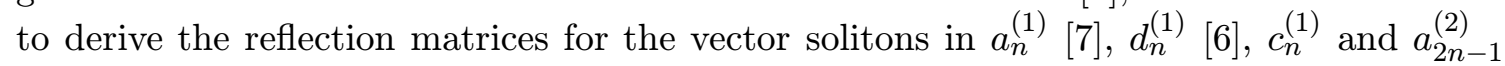
affine Toda theories. In section ' ${ }_{-1}^{\prime} i_{1}^{\prime}$ we show that the half line conserved charges described in sections 零 and are in fact exact $[\underline{\bar{\imath}}]$.

\section{The Yang-Baxter equation and quantum groups}

In this section we describe the Yang-Baxter equation

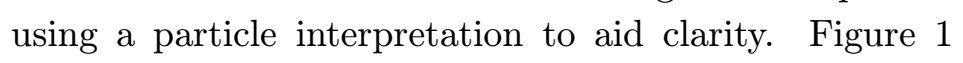
shows two three-particle scattering processes in two dimensions. The two scattering processes differ only in the order in which the individual two-particle scatterings take place. In an integrable theory the scattering amplitude will be independent of this order and this is expressed by

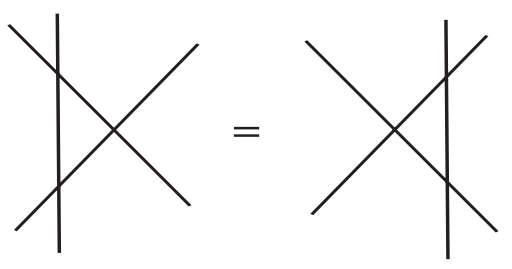

Figure 1: Yang-Baxter equation. the equality in the figure. Each of the lines in the figure stands for a vector space spanned by all the quantum particle states in the same multiplet. Each vector space carries a representation of the symmetry group describing a particle multiplet. Let us denote the three vector spaces by $V_{\theta_{1}}^{\mu}, V_{\theta_{2}}^{\nu}, V_{\theta_{3}}^{\lambda}$. Here $\mu, \nu$ and $\lambda$ label the different multiplets and the $\theta_{i}$ give the rapidities of the particles. Two or more adjacent lines, for example those representing the spaces $V_{\theta_{1}}^{\mu}$ and $V_{\theta_{2}}^{\nu}$, are tensor products of those spaces, $V_{\theta_{1}}^{\mu} \otimes V_{\theta_{2}}^{\nu}$.

Crossing lines in the diagram represent scattering of two particles and can be described by a mapping from the vector space representing the initial two-particle state to the vector 
space representing the final two-particle state,

$$
\check{R}^{\mu \nu}\left(\theta_{1}-\theta_{2}\right): V_{\theta_{1}}^{\mu} \otimes V_{\theta_{2}}^{\nu} \rightarrow V_{\theta_{2}}^{\nu} \otimes V_{\theta_{1}}^{\mu} .
$$

Because of Lorentz invariance the matrix $\check{R}^{\mu \nu}$ depends only on the difference $\theta_{1}-\theta_{2}$ of the spectral parameters. This matrix, describing the scattering of two particles, is usually referred to as the two-particle S-matrix. The equation in figure

$$
\begin{aligned}
\left(\check{R}^{\mu \nu}\left(\theta_{1}-\theta_{2}\right) \otimes 1\right) & \left(1 \otimes \check{R}^{\mu \lambda}\left(\theta_{1}-\theta_{3}\right)\right)\left(\check{R}^{\nu \lambda}\left(\theta_{2}-\theta_{3}\right) \otimes 1\right) \\
= & \left(1 \otimes \check{R}^{\nu \lambda}\left(\theta_{2}-\theta_{3}\right)\right)\left(\check{R}^{\mu \lambda}\left(\theta_{1}-\theta_{3}\right) \otimes 1\right)\left(1 \otimes \check{R}^{\mu \nu}\left(\theta_{1}-\theta_{2}\right)\right) .
\end{aligned}
$$

This is the Yang-Baxter equation. We used the symbol $\check{R}$ to denote the scattering matrix because a solution of the Yang-Baxter equation is conventionally referred to as an $R$-matrix. Further details on $R$-matrices and the Yang-Baxter equation can be found throughout the

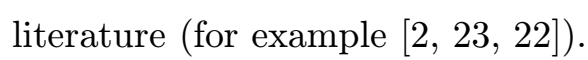

The Yang-Baxter equation (2.2i) is non-linear, and so it is difficult to solve directly. However in the physical context of particle scattering in an integrable theory described above it is possible that the scattering matrix is uniquely determined by the symmetry of the theory. It is then enough to solve the symmetry requirement in order to find a solution of the Yang-Baxter equation. This is of great advantage because the symmetry requirement corresponds to a simple linear equation. The quantum groups generated by non-local conserved charges in certain integrable quantum field theories describe such symmetries. Examples are the Yangians $Y(\mathfrak{g})$ in the principal chiral model and the quantum affine algebras $U_{q}(\hat{\mathfrak{g}})$ in affine Toda field theory.

Let us denote the quantum group by $\mathcal{A}$. The vector spaces $V_{\theta}^{\mu}$ are representations of $\mathcal{A}$, also called $\mathcal{A}$-modules. The tensor product spaces $V_{\theta_{1}}^{\mu} \otimes V_{\theta_{2}}^{\nu}$ formed by the two-particle states should also be $\mathcal{A}$-modules. Therefore $\mathcal{A}$ is required to be a bialgebra for which there is defined a coproduct

$$
\triangle: \mathcal{A} \rightarrow \mathcal{A} \otimes \mathcal{A}
$$

The coproduct encodes how a symmetry charge acts on multiparticle states and in the case of non-local charges this is usually not cocommutative.

Symmetry requires that the scattering matrix $\left(\overline{2} . \overline{1} \overline{1}_{1}\right)$ is an $\mathcal{A}$-module homomorphism, also called an intertwiner. Thus it has the property that for all $Q \in \mathcal{A}$

$$
Q^{\nu \mu} \check{R}^{\mu \nu}=\check{R}^{\mu \nu} Q^{\mu \nu}
$$

where $Q^{\mu \nu}$ denotes the action of $Q$ on $V_{\theta_{1}}^{\mu} \otimes V_{\theta_{2}}^{\nu}$. We want the quantum group symmetry to be strong enough to determine the scattering matrix uniquely. Thus there should be only a unique solution of the intertwining equation (2.2.4). This is ensured by Schur's lemma if the tensor product module $V_{\theta_{1}}^{\mu} \otimes V_{\theta_{2}}^{\nu}$ is irreducible for generic values of the spectral parameters. This is know to be the case for Yangians $Y(\mathfrak{g})$ and quantum affine algebras $U_{q}(\hat{\mathfrak{g}})$.

As stated above, in integrable quantum field theories the scattering matrix $\check{R}^{\mu \nu}$ satisfies the Yang-Baxter equation because the ordering of the two-particle scattering processes does 
not matter. We can now give an independent mathematical reason for why $\check{R}^{\mu \nu}$ satisfies the Yang-Baxter equation. The tensor products $V_{\theta_{1}}^{\mu} \otimes V_{\theta_{2}}^{\nu} \otimes V_{\theta_{3}}^{\lambda}$ are irreducible representations of $\mathcal{A}$, for generic values of the spectral parameters. Thus the two sides of the YangBaxter equation represent two possible maps from one irreducible representation to another. Therefore, by Schur's Lemma, they must be proportional to each other. The constant of proportionality can be shown to be equal to 1 by consideration of the determinants of the two sides of $(2 \overline{2} \cdot 2 \overline{2})$ and the behaviour of the constant in the classical limit [1]

\section{The reflection equation and boundary quantum groups}

Figure $1 \overline{2}$ is shows two different ways in which a twoparticle reflection process can factorize into single-particle reflection and two-particle scattering processes. In integrable quantum field theories the corresponding amplitudes must be equal. The incoming lines represent vector spaces $V_{\theta_{1}}^{\mu}, V_{\theta_{2}}^{\nu}$. As in the Yang-Baxter equation the spaces carry a representation of the symmetry algebra de-

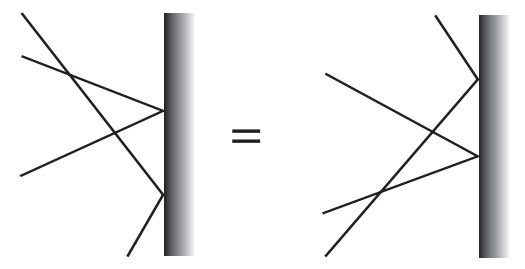

Figure 2: Reflection equation scribing a particle multiplet, labelled by the Greek letters.

The spaces also carry a spectral parameter $\theta_{i}$, giving the rapidity of the corresponding particle. After reflection from the boundary the particles will have reversed their rapidities and thus the outgoing lines represent vector spaces $V_{-\theta_{1}}^{\bar{\mu}}, V_{-\theta_{2}}^{\bar{\nu}}$. The boundary also carries a vector space, $W^{\lambda}$, to describe properties such as boundary spin or boundary bound states. Note that this space does not have a rapidity parameter as the boundary is assumed to be stationary. As in the Yang-Baxter equation adjacent lines, including the boundary, represent tensor products of the relevant vector spaces. The point of contact of the boundary with and incoming and an outgoing line indicates reflection of a particle from the boundary, this process can be described with a mapping similar to $\left(\overline{2} \cdot \overline{1}_{1}^{\prime}\right)$,

$$
K^{\mu \nu}(\theta): V_{\theta}^{\mu} \otimes W^{\lambda} \rightarrow V_{-\theta}^{\bar{\mu}} \otimes W^{\lambda} .
$$

$K^{\mu \nu}$ are the reflection matrices or K-matrices. Intersection of two lines, incoming or outgoing, represents scattering of the particles and, as in the previous section, is represented by $\check{R}$. The equation in figure $\underset{2}{\underline{2}}$ then reads

$$
\begin{aligned}
& \left(\check{R}^{\bar{\nu} \bar{\mu}}\left(\theta_{1}-\theta_{2}\right) \otimes 1\right)\left(I \otimes K^{\mu \lambda}\left(\theta_{1}\right)\right)\left(\check{R}^{\mu \bar{\nu}}\left(\theta_{1}+\theta_{2}\right) \otimes 1\right)\left(I \otimes K^{\nu \lambda}\left(\theta_{2}\right)\right) \\
& \quad=\left(I \otimes K^{\nu \lambda}\left(\theta_{2}\right)\right)\left(\check{R}^{\nu \bar{\mu}}\left(\theta_{1}+\theta_{2}\right) \otimes 1\right)\left(I \otimes K^{\mu \lambda}\left(\theta_{1}\right)\right)\left(\check{R}^{\mu \nu}\left(\theta_{1}-\theta_{2}\right) \otimes 1\right) .
\end{aligned}
$$

This is the reflection equation. Further details on the reflection equation can be found

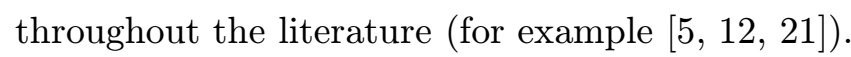

The reflection equation ( $(\overline{3} . \overline{2}-1)$ is non-linear. As so many other similarities hold between this and the Yang-Baxter equation it might be expected that a method analogous to the intertwiners, as described in the previous section, may exist to find $K$-matrices by the solution of a linear equation. To this end we are motivated to find boundary quantum 


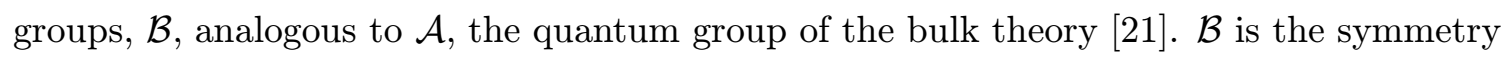
algebra of the boundary theory. It is generated by the non-local charges that are still conserved after the introduction of the boundary. The vector space associated with the boundary $W^{\lambda}$ is a representation of the algebra $\mathcal{B}$, called a $\mathcal{B}$-module. The tensor product $V_{\theta}^{\mu} \otimes W^{\lambda}$, describing a particle and the boundary, must also be a $\mathcal{B}$-module. We know that the space $V_{\theta}^{\mu}$ is an $\mathcal{A}$-module so $\mathcal{B}$ is required to be an $\mathcal{A}$ coideal, for which there exists a coproduct,

$$
\triangle: \mathcal{B} \rightarrow \mathcal{A} \otimes \mathcal{B}
$$

This coproduct describes the action of the non-local charges on states containing particles and the boundary. This observation explains why the symmetry algebra of an integrable quantum field theory with a boundary does not have to be a bialgebra: the particles away from the boundary still transform in representations of the symmetry of the theory on the whole line.

Just as the $R$-matrix was an $\mathcal{A}$-module homomorphism it is now clear that the symmetry requires that the $K$-matrix is a $\mathcal{B}$-module homomorphism, or intertwiner. So for all $\hat{Q} \in \mathcal{B}$ the $K$-matrix has to satisfy

$$
\hat{Q}^{\bar{\mu} \lambda} K^{\mu \lambda}=K^{\mu \lambda} \hat{Q}^{\mu \lambda}
$$

where $\hat{Q}^{\mu \lambda}$ denotes the action of $\hat{Q}$ on $V_{\theta}^{\mu} \otimes W^{\lambda}$.

If $V_{\theta_{1}}^{\mu} \otimes V_{\theta_{2}}^{\nu} \otimes W^{\lambda}$ are irreducible representations, for generic $\theta_{i}$, then by Schur's Lemma a solution of the linear intertwining equation (13.4) is also a solution of the reflection equation $(132)$. The argument is exactly analogous to the one presented in the previous section to show that a solution of eq. (12.4) is a solution of the Yang-Baxter equation.

The problem of finding reflection matrices has now been reduced to finding the boundary quantum group, and the solution of the linear equation (3).4. The following properties have to hold in order for $\mathcal{B}$ to lead us to solutions of the reflection equation:

- $\mathcal{B}$ must be an $\mathcal{A}$ coideal,

- Intertwiners $K^{\mu \lambda}(\theta): V_{\theta}^{\mu} \otimes W^{\lambda} \rightarrow V_{-\theta}^{\bar{\mu}} \otimes W^{\lambda}$ must exist,

- the tensor product representations $V_{\theta_{1}}^{\mu} \otimes V_{\theta_{2}}^{\nu} \otimes W^{\lambda}$ must be irreducible for generic values of the spectral parameters $\theta_{i}$.

We will now go on to find such boundary quantum groups by studying the symmetries in concrete integrable quantum field theories on the half-line.

\section{Sine-Gordon field on the half line}

We seek to find the boundary quantum group for the sine-Gordon field restricted to the half line by an integrable boundary condition. Initially, however, we consider the whole 
line theory. It is useful to view the sine-Gordon model as a perturbation of the free bosonic field theory, with action $\left[2 \overline{2} \overline{2}_{-1}^{\prime}, \overline{\underline{3}}_{1}\right]{ }^{2}$

$$
S=\frac{-1}{8 \pi} \iint\left(\left(\partial_{x} \phi\right)^{2}+\left(\partial_{t} \phi\right)^{2}\right) d x d t-\Phi_{\mathrm{bulk}}^{\mathrm{pert}}
$$

where

$$
\Phi_{\mathrm{bulk}}^{\mathrm{pert}}=\frac{\lambda}{4 \pi} \iint\left(e^{i \hat{\beta} \phi(x, t)}+e^{-i \hat{\beta} \phi(x, t)}\right) d x d t .
$$

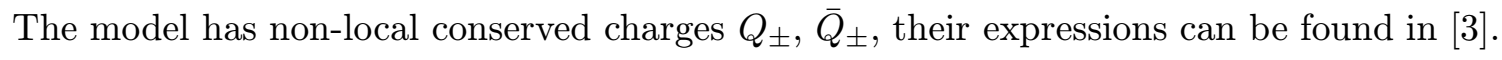
These charges together with the Lorentz boost $D$ and the topological charge

$$
T=\frac{\hat{\beta}}{2 \pi} \int \partial_{x} \phi d x
$$

generate the quantum affine algebra $U_{q}\left(\widehat{s l_{2}}\right)$ with zero center.

The action of the non-local conserved charges on two-soliton states are given by the coproduct

$$
\begin{aligned}
& \triangle\left(Q_{ \pm}\right)=Q_{ \pm} \otimes I+q^{ \pm T} \otimes Q_{ \pm}, \\
& \triangle\left(\bar{Q}_{ \pm}\right)=\bar{Q}_{ \pm} \otimes I+q^{\mp T} \otimes \bar{Q}_{ \pm}, \\
& \triangle(T)=T \otimes I+I \otimes T,
\end{aligned}
$$

where

$$
q=e^{2 i \pi\left(1-\hat{\beta}^{2}\right) / \hat{\beta}^{2}} .
$$

We will treat the sine-Gordon model on the half-line with general integrable boundary conditions as a perturbation of the free field theory on the half line with Neumann boundary condition $\left.\partial_{x} \tilde{\phi}\right|_{x=0}=0$. Note that to avoid confusion we decorate the fields in the theory on the half-line with a tilde. The Neumann boundary condition selects among all possible solutions on the whole line exactly those that are invariant under parity $\mathcal{P}: x \mapsto-x$. We find that the easiest way to do quantum calculations in the theory with Neumann boundary condition is to view it as the parity invariant subsector of the theory on the whole line.

The perturbing term $\Phi_{\text {bulk }}^{\text {pert }}$ in ( $\left(\overline{4} . \overline{2}_{i}^{\prime}\right)$ is invariant under parity transform, $\mathcal{P}$, and so we can also use it as the perturbing term to obtain the sine-Gordon model on the half-line with Neumann boundary condition.

The whole line conserved charges transform under parity as

$$
\mathcal{P}: T \mapsto-T, \quad \mathcal{P}: Q_{ \pm} \mapsto \bar{Q}_{\mp}, \quad \mathcal{P}: \bar{Q}_{ \pm} \mapsto Q_{\mp} .
$$

The parity invariant combinations $\tilde{Q}_{ \pm}=Q_{ \pm}+\bar{Q}_{\mp}$ are well defined in the half-line theory and are conserved [īi]

\footnotetext{
${ }^{2}$ We use the conventions of $[3 i$. The contribution to these proceedings by Bajnok, Palla, and Takács on the boundary sine-Gordon model [i]1] uses more standard conventions. The relations are that $\hat{\beta}=\beta / \sqrt{4 \pi}$, $\phi=\sqrt{4 \pi} \Phi$, and $\lambda=2 \pi m^{2} / \beta^{2}$.
} 
We now replace the Neumann condition with the more general integrable boundary

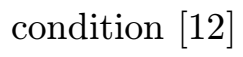

$$
\left.\partial_{x} \tilde{\phi}(x, t)\right|_{x=0}=i \hat{\beta} \lambda_{b}\left(\epsilon_{-} e^{i \hat{\beta} \tilde{\phi}(0, t) / 2}-\epsilon_{+} e^{-i \hat{\beta} \tilde{\phi}(0, t) / 2}\right)
$$

where $\epsilon_{+}$and $\epsilon_{-}$are two arbitrary boundary parameters ${ }^{3}$. We treat this as a boundary perturbation on the Neumann half line problem discussed above, with action $S_{\epsilon}$ given by

$$
S_{\epsilon}=S_{0}+\Phi_{\text {bound }}^{\text {pert }}
$$

where $S_{0}$ is the action of the Neumann boundary theory and

$$
\Phi_{\text {bound }}^{\text {pert }}=\frac{\lambda_{b}}{2 \pi} \int\left(\epsilon_{-} e^{i \hat{\beta} \tilde{\phi}(0, t) / 2}+\epsilon_{+} e^{-i \hat{\beta} \tilde{\phi}(0, t) / 2}\right) d t
$$

We can then use first order boundary perturbation theory [i $[\overline{2} \overline{2}, \overline{2}, \overline{2} \overline{0}]$ to find the half line

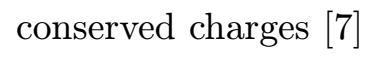

$$
\hat{Q}_{ \pm}=Q_{ \pm}+\bar{Q}_{\mp}+\hat{\epsilon}_{ \pm} q^{ \pm T}
$$

where

$$
\hat{\epsilon}_{ \pm}=\frac{\lambda_{b} \epsilon_{ \pm} \hat{\beta}^{2}}{2 \pi\left(1-\hat{\beta}^{2}\right)} .
$$

These expressions were first conjectured in [i $\left[\begin{array}{l}1 \\ \mathbf{6}\end{array}\right]$. We denote the symmetry algebra generated by these charges by $\mathcal{B}_{q}^{\epsilon}\left(\widehat{s l_{2}}\right) . \mathcal{B}_{q}^{\epsilon}\left(\widehat{s l_{2}}\right)$ is a coideal subalgebra of $U_{q}\left(\widehat{s l_{2}}\right)$ because

$$
\triangle\left(\hat{Q}_{ \pm}\right)=\hat{Q}_{ \pm} \otimes I+q^{ \pm T} \otimes\left(\hat{Q}_{ \pm}-\hat{\epsilon}_{ \pm} I\right) \in U_{q}\left(\widehat{s l_{2}}\right) \otimes \mathcal{B}_{q}^{\epsilon}\left(\widehat{s l_{2}}\right)
$$

This boundary quantum group can now be used to calculate reflection matrices by solving the intertwining relation (3) $\left({ }^{3}\right)$. Choosing the representation $W^{\lambda}$ to be the trivial representation (corresponding to the boundary ground state) and the representation $V^{\mu}$ to be the spin $1 / 2$ representation (in which the sine-Gordon solitons are known to transform) one rederives the known sine-Gordon soliton reflection matrix of Ghoshal and Zamolod-

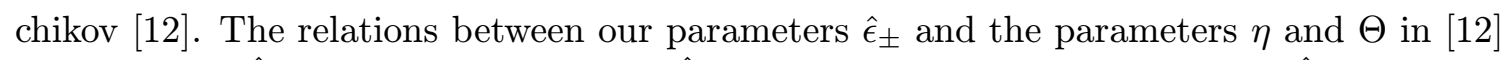
are $\hat{\epsilon}_{ \pm}=f(\lambda, \hat{\beta}) \cos (\eta \pm i \Theta)$ where $f(\lambda, \hat{\beta})$ is a complicated function of $\lambda$ and $\hat{\beta}$. By taking the representation $V^{\mu}$ to be higher spin representations of $U_{q}\left(\widehat{s l_{2}}\right)$ one obtains new $s l_{2}$ $K$-matrices $[\overline{\underline{9}} \overline{1}]$.

\section{Affine-Toda fields on the half line}

We will now follow a similar procedure to find boundary quantum groups $\mathcal{B}_{q}^{\epsilon}(\hat{\mathfrak{g}})$ for every affine Lie algebra $\hat{\mathfrak{g}}$ by deriving the non-local symmetry charges in affine Toda field theories.

\footnotetext{
${ }^{3}$ These are related to the boundary parameters $M_{0}$ and $\varphi_{0}$ used in [in $]$ by $\exp \left(i \beta \varphi_{0}\right)=\epsilon_{+} / \epsilon_{-}$and $M_{0}^{2}=\left(\lambda_{b} / \pi\right)^{2} \epsilon_{+} \epsilon_{-}$.
} 
For every affine Lie algebra $\hat{\mathfrak{g}}$ of rank $n$ there is an affine Toda field theory describing a $n$-component bosonic field in $1+1$ dimensions with action [i $\overline{1} \overline{7}$ ]

$$
S=\frac{-1}{8 \pi} \iint\left(\left(\partial_{x} \phi\right)^{2}+\left(\partial_{t} \phi\right)^{2}\right) d x d t-\Phi_{\text {bulk }}^{\mathrm{pert}}
$$

where

$$
\Phi_{\mathrm{bulk}}^{\mathrm{pert}}=\frac{\lambda}{4 \pi} \iint\left(\sum_{j=0}^{n} e^{-i \beta \alpha_{j} \cdot \phi /\left|\alpha_{j}\right|^{2}}\right) d x d t .
$$

The $\alpha_{j}$ are the simple roots of $\hat{\mathfrak{g}}$ projected onto the root space of $\mathfrak{g}$, the finite dimensional Lie algebra. The theory has non-local conserved charges $Q_{j}$ and $\bar{Q}_{j}$ for $j=0,1, \ldots, n$ [i]j]. These, along with the Lorentz boost $D$ and the topological charges

$$
T_{j}=\frac{\beta}{2 \pi} \int \alpha_{j} \cdot \partial_{x} \phi d x
$$

generate the quantum affine algebra $U_{q}(\hat{\mathfrak{g}})$ with zero centre [i] $\mathbf{I}_{-}^{1} \overline{1}$. The non-local charges act on two-soliton states through the coproduct

$$
\begin{aligned}
& \triangle\left(Q_{j}\right)=Q_{j} \otimes I+q^{T_{j}} \otimes Q_{j}, \\
& \triangle\left(\bar{Q}_{j}\right)=\bar{Q}_{j} \otimes I+q^{T_{j}} \otimes \bar{Q}_{j}, \\
& \triangle\left(T_{j}\right)=T_{j} \otimes I+I \otimes T_{j} .
\end{aligned}
$$

For $\hat{\mathfrak{g}}=\widehat{s l}_{2}$ the affine Toda theory becomes the sine-Gordon model and the conserved

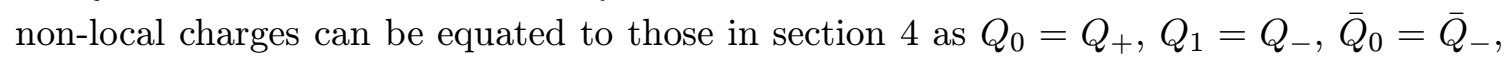
$\bar{Q}_{1}=\bar{Q}_{+}$and $T_{0}=-T_{1}=T$.

We are interested in restricting the field, for simply laced $\hat{\mathfrak{g}}$, to the half line with the boundary condition

$$
\left.\partial_{x} \tilde{\phi}(x, t)\right|_{x=0}=-i \beta \lambda_{b} \sum_{j=0}^{n} \epsilon_{j} \alpha_{j} e^{-i \beta \alpha_{j} \cdot \tilde{\phi}(0, t) / 2},
$$

where the $\epsilon_{j}$ are $n+1$ free boundary parameters. It is known that these boundary conditions preserve the integrability of the theory only if all $\left|\epsilon_{j}\right|=1$ or if all $\epsilon_{j}=0$ [i i i ] .

As for the sine-Gordon field we define the Neumann half line theory using parity invariance. We then add a boundary perturbation giving the action,

$$
S_{\epsilon}=S_{0}+\Phi_{\text {bound }}^{\text {pert }}
$$

where $S_{0}$ is the action of the Neumann theory and

$$
\Phi_{\text {bound }}^{\text {pert }}=\frac{\lambda_{b}}{2 \pi} \int\left(\sum_{j=0}^{n} \epsilon_{j} e^{-i \beta \alpha_{j} \cdot \tilde{\phi}(0, t) / 2}\right) d t .
$$


Using first order boundary perturbation theory the non-local conserved charges have been found to be [i]i]]

$$
\hat{Q}_{j}=Q_{j}+\bar{Q}_{j}+\hat{\epsilon}_{j} q_{j}^{T_{j}}
$$

where

$$
\hat{\epsilon}_{j}=\frac{\lambda_{b} \hat{\beta}^{2}}{2 \pi\left(1-\hat{\beta}^{2}\right)} \epsilon_{j}
$$

While we have performed the calculation only in the case of simply laced $\hat{\mathfrak{g}}$, we believe the expressions ( $(\overline{5}-\overline{3})$ to be correct in all cases.

We denote the symmetry algebra generated by the charges $\hat{Q}_{j}, j=0,1, \ldots, n$ by $\mathcal{B}_{q}^{\epsilon}(\hat{\mathfrak{g}}) \subset U_{q}(\hat{\mathfrak{g}})$. In [iㅎ] we introduced the name 'quantum affine reflection algebras' for these algebras to express that they are subalgebras of quantum affine algebras which at the same time are reflection equation algebras in the sense of Sklyanin [2] $\left.\overline{1}_{-}\right]$. The algebra $\mathcal{B}_{q}^{\epsilon}(\hat{\mathfrak{g}})$ is a coideal subalgebra of $U_{q}(\hat{\mathfrak{g}})$ because

$$
\triangle\left(\hat{Q}_{j}\right)=\hat{Q}_{j} \otimes I+q^{T_{j}} \otimes\left(\hat{Q}_{j}-\hat{\epsilon}_{j} I\right) \in U_{q}(\hat{\mathfrak{g}}) \otimes \mathcal{B}_{q}^{\epsilon}(\hat{\mathfrak{g}}),
$$

as can easily be checked using ( $(\overline{5}-4)$.

To first order in boundary perturbation theory the charges $\hat{Q}_{j}$ are conserved for arbitrary values of the boundary parameters $\epsilon_{j}$. However we found that the intertwining relation ( $(\overline{3} .4)$ does not always have a solution. In fact we found that for simply laced $\hat{\mathfrak{g}}$ the boundary parameters $\hat{\epsilon}_{j}$ are fixed up to sign by the requirement that a reflection matrix for the vector representation should exist. This is the quantum generalization of the result of [i] which showed that the boundary condition preserves classical integrability only if the boundary parameters $\epsilon_{j}$ are fixed up to sign. For non-simply laced $\hat{\mathfrak{g}}$ we find that some of the parameters $\hat{\epsilon}_{j}$ can be free and this again parallels the classical results of [i]山.

The boundary quantum groups $\mathcal{B}_{q}^{\epsilon}(\hat{\mathfrak{g}})$ have been successfully used to rederive the vector representation reflection matrices for $a_{n}^{(1)}$ affine Toda theories [i] unknown reflection matrices for $d_{n}^{(1)}[\dot{6} \bar{i}], c_{n}^{(1)}$ and $a_{2 n-1}^{(2)}$ affine Toda theories.

\section{Non-perturbative derivation of non-local conserved charges}

In sections found using first order boundary perturbation theory. One might be worried that at higher order perturbation theory additional terms could appear in the expression for the half line conserved charges. The fact that the charges have already been used successfully to derive new $K$-matrices for the vector representation does not rule out the presence of additional terms because these terms could vanish in the vector representations. We can, however, derive the expression for the charges also in a non-perturbative way by using the vector soliton reflection matrix. This derivation, which we will present briefly in this section,

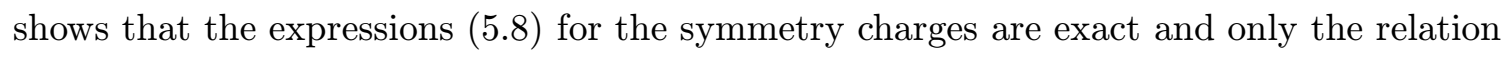


$(1,59)$ between the bare and the renormalized boundary parameters may receive higher order corrections $[\underline{\bar{i}}]$.

Using the vector soliton reflection matrix $K^{\mu}(\theta)$ we construct a matrix

$$
B_{\theta}^{\mu}=\bar{L}_{\theta}^{\bar{\mu}}\left(K^{\mu}(\theta) \otimes 1\right) L_{\theta}^{\mu} \in \operatorname{Hom}\left(V_{\theta}^{\mu}, V_{-\theta}^{\bar{\mu}}\right) \otimes U_{q}(\hat{\mathfrak{g}}),
$$

with

$$
\begin{aligned}
& L_{\theta}^{\mu}=\left(\pi_{\theta}^{\mu} \otimes i d\right)(\mathcal{R}(x)) \in \operatorname{End}\left(V_{\theta}^{\mu}\right) \otimes U_{q}(\hat{\mathfrak{g}}), \\
& \bar{L}_{\theta}^{\bar{\mu}}=\left(\pi_{-\theta}^{\bar{\mu}} \otimes i d\right)\left(\mathcal{R}^{o p}(x)\right) \in \operatorname{End}\left(V_{-\theta}^{\bar{\mu}}\right) \otimes U_{q}(\hat{\mathfrak{g}}),
\end{aligned}
$$

where $\pi_{\theta}^{\nu}$ denotes the representation $\pi_{\theta}^{\nu}: U_{q}(\hat{\mathfrak{g}}) \rightarrow \operatorname{End}\left(V_{\theta}^{\nu}\right), \mathcal{R}(x)=\left(\Psi_{x} \otimes i d\right)(\mathcal{R}), \Psi_{x}$ is a homomorphism defined by $\Psi_{x}\left(Q_{j}\right)=x Q_{j}, \Psi_{x}\left(\bar{Q}_{j}\right)=x^{-1} \bar{Q}_{j}, \Psi_{x}\left(T_{j}\right)=T_{j}, \mathcal{R}$ is the universal $R$-matrix [14i] and $\mathcal{R}^{o p}$ is the opposite universal $R$-matrix found by swapping the tensor factors in $\mathcal{R}$. It can now be shown that any $K$-matrix that satisfies the reflection

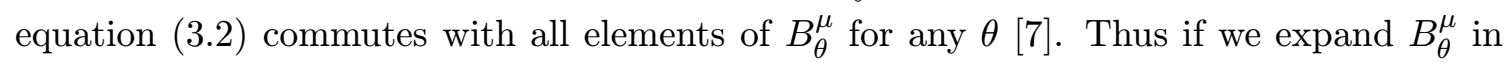
terms of the spectral parameter, then every term in the expansion will commute with any $K$-matrix. Thus every term in the expansion is a symmetry charge. In the cases of $\hat{\mathfrak{g}}=a_{n}^{(1)}$ and $\hat{\mathfrak{g}}=d_{n}^{(1)}$ we have checked explicitly that the first term in this expansion reproduces exactly the charges $\left(\begin{array}{l}5.8 \\ -5\end{array}\right)$ derived previously.

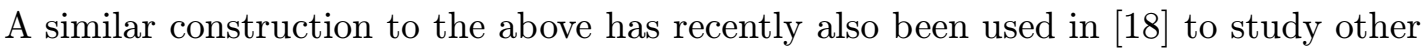
properties of the boundary quantum groups.

\section{Summary and Outlook}

We have presented a brief review of the Yang-Baxter equation describing how the concept of quantum group arises and how solutions of the Yang-Baxter equation can be found as intertwiners of representations of that group. We also presented a review of the reflection equation and introduced the concept of a boundary quantum group as a coideal subalgebra of the quantum group. We showed that solutions to the reflection equation can be found as intertwiners of representations of the boundary quantum group. We showed how the conserved non-local charges for affine Toda theories on the half line can be found using first order perturbation theory, and how these generate boundary quantum groups. Finally we showed that these conserved charges are exact.

There is much work remaining in this area, reflection matrices for the vector representations of $b_{n}^{(1)}, a_{2 n}^{(2)}, d_{n+1}^{(2)}$ and the exceptional algebras have yet to be found. Boundary quantum groups corresponding to Dirichlet type boundary conditions need to be studied [1] $\overline{1} \overline{1}]$, as do higher representations of the presently known groups.

\section{References}

[1] Bajnok, Z., Palla, L., Takacs, G., Boundary sine-Gordon model, hep-th/0211132].

[2] Baxter, R. J., Exactly solved models in statistical mechanics, 1982, (Academic Press Inc.).

[3] Bernard, D., Leclair, A., Quantum group symmetries and nonlocal currents in 2-D QFT, 'Comm. Math Phys. $142 \overline{2}(1991) 991$. 
[4] Bowcock, P., Corrigan, E. F., Dorey, P. E., Rietdijk, R. H., Classically integrable boundary conditions for affine Toda field theories, №cl. Phys. B 445_(1995) 469. [hep-th/9501098].

[5] Cherednik, I. V., Factorizing particles on a half line and root systems, Theor. Math. Phys. $\mathbf{6} \overline{\mathbf{1}}$, - - -

[6] Delius, G. W., George, A., Quantum affine reflection algebras of type $d_{n}^{(1)}$ and reflection matrices, to appear in Lett. Math. Phys., [math.QA/0208043].

[7] Delius, G. W., MacKay, N. J., Quantum group symmetry in sine-Gordon and affine Toda field theories on the half-line, to appear in Commun. Math. Phys., [hep-th/0112023].

[8] Delius, G. W., MacKay, N. J., Short, B. J., Boundary remnant of Yangian symmetry and the structure of rational reflection matrices, Phhys. Lett. B $\mathbf{5 2 2}(2001)$ 335. [hep-th/010911

[9] Delius, G. W., Nepomecchie, R. I., Solutions of the boundary Yang-Baxter equation for

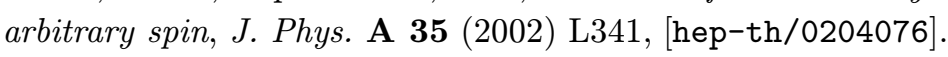

[10] Drinfeld, V. G., Hopf algebras and the quantum Yang-Baxter equation, 'Sov. Math. Dokil. $\overline{3} \overline{2}$ ' - -

[11] Felder, G., Leclair, A., Restricted quantum affine symmetry of perturbed minimal conformal models, Int. J. Mod. Phys. A $\mathbf{7}(19 \overline{9} 2) 239$, [hep-th/9109019].

[12] Ghoshal, S., Zamolodchikov, A. B., Boundary S matrix and boundary state in

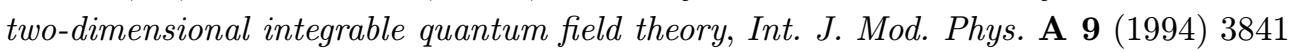
$[$ hep-th/9306002i].

[13] Jimbo, M. Quantum $R$ matrix for the generalized Toda system, (1986) $53 \overline{7}$

[14] Khoroshkin, S. M., Tolstoy, V. N., The universal R-matrix for quantum untwisted affine Lie algebras, 'Eunct. Anal. Áppl. 26 - 1992$)$ - 69 .

[15] MacKay, N., Short, B., Boundary scattering in the principal chiral model, [hep-th/0107256].

[16] Mezincescu, L., Nepomechie, R. I., Fractional-spin integrals of motion for the boundary sine-Gordon model at the free fermion point, 'Int. [hep-th/9709078i].

[17] Mikhailov, A. V., Olshanetsky, M. A., Perelomov, A. M., Two-dimensional generalized Toda lattice, 'Comm. Math. Phys. $\mathbf{7 9} \mathbf{9}(1981) 473$.

[18] Molev, A. I., Ragoucy, E., Sorba, P., Coideal subalgebras in quantum affine algebras, [math.QA/0208140].

[19] Nepomechie, R.I., Boundary quantum group generators of type A, [hep-th/0204181].

[20] Penati, S., Zanon, D., Quantum integrability in two-dimensional systems with boundary, Phys. Lett. B-358 (1995) 63

[21] Skilyanin, E. K., Boundary conditions for integrable quantum systems, "IJ. Phys. i - - - 2375 .

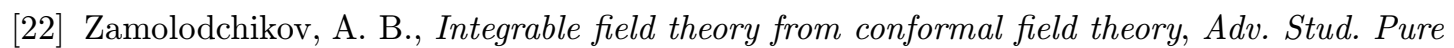
:

[23] Zamolodchikov, A. B., Zamolodchikov, A. B., Factorized S-matrix in two dimensions as the

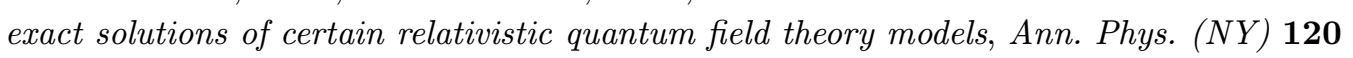
: - - $(1979)-25 \overline{3}$ 